\title{
Sex ratio equilibrium under partial sib mating
}

\author{
Peter D. Taylor
}

Department of Mathematics and Statistics, Queen's University, Kingston, Ontario, K7L 3N6.

\begin{abstract}
A general equation for equilibrium sex allocation is provided which models asymmetries between son and daughter in the costs of offspring production, the genetic relatedness to the controlling genotype and the amount of competition for mating opportunities and reproductive resources. A genetic proof is given which is valid for diploid and haplodiploid systems with a variety of modes of sex allocation control, provided sib mating is the only source of inbreeding. By way of example, some variations of Hamilton's (1967) model are discussed.
\end{abstract}

\section{INTRODUCTION}

Hamilton (1967 and 1979) discussed a sex allocation model in which there was partial sib-mating and a variety of genetic sex ratio control mechanisms. My purpose is to provide a condition (3) for equilibrium sex allocation which generalises both his work and the standard Shaw-Mohler (1953) formula

$$
\frac{d f}{f}+\frac{d m}{m}=0
$$

Roughly speaking, the equilibrium equation is that which would be obtained from a non-genetic, game-theoretic analysis of the problem, using relatedness coefficients. Indeed I will point out that if $w$ is the fitness function belonging to such a model then the equilibrium equation is simply the condition, $d w=0$, that $w$ be stationary. The equilibrium condition allows identification of the three principal factors which cause sex ratio bias, these being son-daughter asymmetries in 1) offspring production, 2) offspring genotype, 3) breeding opportunities. The magnitude of the last two may be affected by the level of inbreeding, but inbreeding per se has no effect on sex ratio equilibrium. I provide a precise genetic proof of the result. I do not consider in this paper the question of stability.

\section{Population structure}

Suppose we have an infinite population with discrete, non-overlapping generations, and two types of individuals, female and male. I assume diploid genetics with the standard X-Y sex determining system. [The important phenomenon of haplodiploidy is obtainable by restricting attention to the X-chromosome.]

I assume the life cycle has two stages. In stage one, offspring are produced by what I will call mated females. Genetically, this term refers to the combined (tetraploid) genotype of female and mate. Each mated female produces $f$ daughters and $m$ sons where there is a negative tradeoff between $f$ and $m$. A simple way to model this is with a parameter $r, 0 \leqq r \leqq 1$, which can be thought of as the proportion of reproductive energy allocated to production of sons. Then $f=f(r)$ and $m=m(r)$ and the feasible pairs $(f, m)$ lie on a tradeoff curve in $f-m$ space. The classic case considered by Fisher (1930) of a linear tradeoff curve is $f=1-r, m=r$.

In stage two, the offspring generation mates to produce next generation mated females. I denote by $P$ the probability that a female at the end of stage one breeds, and by $Q$ the expected number of mates which breed of a stage one male. Then $Q=P f / m$ if the whole population employs the allocation $(f, m)$. For simplicity I assume a mated female has only one mate, but this restriction is lifted in the discussion.

I assume that a female chooses her mate either from her brothers or at random in the population. Thus sib-mating is the only source of inbreeding. I assume offspring are made from the mated female genotype in the standard way: one haploid gamete from each parent. Thus our assumptions rule out more general forms of inbreeding, such as first 
cousin or father-daughter, and such phenomena as laying workers.

\section{Control of sex allocation}

The value of the allocation parameter $r$ employed by a mated female is determined as follows. She first manufactures a control genotype using genetic material of herself and her mate, following fixed selection rules, and the value of $r$ is then determined by the genotype of the control individual at a single locus, which we refer to as the control locus, which may be autosomal or sex-linked. The control genotype may be simply one of the parents, or may be a duaghter or a son or some probabilistic mixture of these. For example, to model the much studied phenomenon of worker control of sex ratio in a haplodiploid population, we take a daughter as the control genotype and the control locus to be on the X-chromosome. We will assume the control genotype is either haploid or diploid.

1 denote by $R_{f}$ and $R_{m}$ the specific (to the control locus) relatedness of the control genotype to the daughters and sons respectively of the mated female. That is, $R_{f}$ is the expected number of copies (identical by descent) in a daughter, of a random allele at the control locus in the control genotype, and $R_{m}$ is the same for a son. $R_{f}$ and $R_{m}$ depend on the level of inbreeding.

These relatedness coefficients are not in standard usage, but they are the most natural objects for our model. They are closely related to Falconer's (1960) coancestry, the probability that two alleles drawn at random from each of two individuals are identical by descent. If we let $c_{f}$ and $c_{m}$ be the specific (to the control locus) coancestry of the control genotype and a daughter and son respectively, then $R_{f}=c_{f} \pi_{f}$ and $R_{m}=c_{m} \pi_{m}$ where $\pi_{f}$ and $\pi_{m}$ are the specific (at the control locus) ploidies of female and male.

\section{The inbreeding coefficient}

Define the inbreeding coefficient $F$ to be the probability that uniting gametes are identical by descent at a random locus. I remark that for partial sibmating, it does not matter whether the locus in question is autosomal or X-linked; in both cases $F=s /(4-3 s)$ where $s$ is the probability of sibmating ( $\mathrm{Li} \mathrm{1955).} \mathrm{At} \mathrm{equilibrium} F$ is also the probability that the two alleles at a random (autosomal or X-linked) locus in a diploid individual are identical by descent.

As an example of the relatedness coefficients, consider the case of $\mathrm{X}$-linked control (isomorphic to haplodiploidy). A standard calculation shows

$$
R_{f}=(3 F+1) / 2 \quad R_{m}=(F+1) / 2
$$

maternal or son control

$$
\begin{array}{ll}
R_{f}=1+F & R_{m}=F \\
& \text { paternal control } \\
R_{f}=(5 F+3) / 4 & R_{m}=(3 F+1) / 4 \\
& \text { daughter control }
\end{array}
$$

Mother and son control are equivalent here, since an $\mathrm{X}$-allele in a son is the same as a random $\mathrm{X}$-allele in the mother. The daughter coefficients are straight averages of the previous two, since a random $\mathrm{X}$ allele in a daughter is in mother or father each with probability $1 / 2$.

It is of interest to observe the effect of $F$ on the $R_{f} / R_{m}$ ratio. In the case of control by the mother, the ratio is near 1 for small $F$ and increases to 2 at $F=1$. For control by father or daughter, the ratio is larger for small $F$ and decreases to 2 at $F=1$.

\section{The equilibrium equation}

Suppose the entire population is employing a certain allocation $r$ which is an interior point of the range $[0,1]$. The equilibrium condition (3) is a necessary condition for this strategy to be evolutionarily stable, that is, resistant to invasion by any rare mutant strategy $r^{\prime}$. It is a local condition, assuming not only that $r^{\prime}$ is rare, but that it is close to $r .1$ formulate the condition in differential form.

Specifically, I assume a single wild type allele at the control locus coding for strategy $r$, and a rare mutant allele which codes for $r^{\prime}$. I consider $r$ fixed and imagine $r^{\prime}$ to be variable, and near $r$. The case $r^{\prime}=r$, for which the mutation causes no phenotypic change, will be important.

A mated female will be called mutant if she has at least one mutant allele in her (tetraploid) genotype, but she may or may not employ strategy $r^{\prime}$, depending on the control genotype. If she does use $r^{\prime}$, she will be called deviant. In this case she makes $f^{\prime}$ daughters and $m^{\prime}$ sons, and I will denote by $P^{\prime}$ the probability that each of her daughters breed and by $Q^{\prime}$ the expected number of breeding mates of each of her sons. In general $P^{\prime}$ and $Q^{\prime}$ will depend on both $r^{\prime}$ and $r$.

To get the equilibrium condition, let $r^{\prime}$ be a differential change from $r, r^{\prime}=r+d r$, and let $d f$, $d m, d P$ and $d Q$ be the corresponding differential changes of $f^{\prime}, m^{\prime}, P^{\prime}$ and $Q^{\prime}$ from $f, m, P$ and $Q$. 
Note that $d P$ and $d Q$ measure the differential increase in breeding probability of a daughter and number of breeding mates of a son of a deviant female.

Then the equilibrium equation, which is derived in the Appendix, is

$$
R_{f}\left(\frac{d f}{f}+\frac{d P}{P}\right)+R_{m}\left(\frac{d m}{m}+\frac{d Q}{Q}\right)=0 .
$$

I emphasize this is a necessary condition for an evolutionarily stable interior value of the sex allocation parameter $r$. In practice it allows us to locate all interior stationary points, and stability is then checked separately.

Let me remark that in a game-theoretic model we would try to write down an expression for the fitness $w^{\prime}$ of the control of a deviant female, and the most obvious candidate would be $w^{\prime}=R_{f} f^{\prime} P^{\prime}+$ $R_{m} m^{\prime} Q^{\prime}$. Then, noting that $f P=m Q,(3)$ is just the condition $d w=0$.

\section{Three factors causing sex ratio bias}

The objective of the evolutionary game is to project genes into future generations, and in a sexual population, one has a choice between placing ones genes, initially, in daughters or sons. Sex ratio bias may be caused by any son-daughter asymmetry in this process. In this model, I class the asymmetries which lead to sex ratio bias into three types.

The first type belongs to stage one and is reflected by asymmetry in the $f-m$ tradeoff curve (about the line $f=m$ ). This type is accounted for already in the Shaw-Mohler equation (1). The classic linear tradeoff $f=1-r, m=r$, is symmetric and leads to a ratio of $r=1 / 2$.

The second type of asymmetry is genetic and is tied to the locus of control. In our equilibrium equation (3) this asymmetry is measured by the relative size of the relatedness coefficients $R_{f}$ and $R_{m}$. For example, when the control locus is autosomal, $R_{f}=R_{m}$ regardless of control genotype, and (3) becomes

$$
\left(\frac{d f}{f}+\frac{d P}{P}\right)+\left(\frac{d m}{m}+\frac{d Q}{Q}\right)=0 .
$$

But sex chromosomes are put into sons and daughters in different quantities, and the asymmetries resulting from sex-linked control are reflected in the $R$ values. The size of this effect depends on the level of inbreeding, sometimes increasing the bias, sometimes not, as we can see in (2). For example if $R_{f}>R_{m}$ (as is the case for $\mathrm{X}$-linked control), a simple geometric argument shows that the equilibrium point of (3) is shifted away from that of (4) towards higher $f$ values, for most tradeoff curves, certainly for those which are linear $(d f / d m=$ constant $)$ or concave-down $(d f / d m$ decreasing with $m)$.

An important special case of X-control is, as I have pointed out, haplodiploidy. If there is no inbreeding then under maternal control $R_{f}=R_{m}$ and under daughter ("worker") control $R_{f}=3 R_{m}$. But if there is some inbreeding then these relationships change as in (2).

The third type of asymmetry belongs to the mating and breeding phase, and measures sex differences in breeding opportunities. This factor is measured by $d P / P$ and $d Q / Q$ and may be referred to as local mate competition or more generally, local (reproductive) resource competition. If all females mate and if males disperse to mate, then $d P=0=d Q$ and (3) beomes

$$
R_{f} \frac{d f}{f}+R_{m} \frac{d m}{m}=0 .
$$

But if a shift in allocation towards sons, of a deviant female, results in fewer mates per son, then $d Q / d m<0$, and (3) will yield a higher $f$ value than (5). Similarly, if a shift towards daughters results in fewer breeding opportunities for each daughter, then $d P / d f<0$, and (3) will yield a higher $m$ value than (5).

\section{Example: variations on Hamilton's model}

To calculate $Q^{\prime}$ we need to know the mating structure; it is not enough to know the level of inbreeding. For example in Hamilton's (1967) model, $N$ unrelated mated females colonise a patch and produce offspring who mate at random before the females disperse to find new colonies. Then a deviant mated female will be the only one in her patch, and, assuming all females mate $\left(P^{\prime}=P=1\right)$ the number of mates of each of her sons is

$$
Q^{\prime}=\frac{(N-1) f+f^{\prime}}{(N-1) m+m^{\prime}},
$$

and $Q=f / m$, the female/male ratio. Note that $Q^{\prime}$ depends on both $r^{\prime}$ and $r$. Taking differentials $\left(r^{\prime}=r+d r\right)$ we get

$$
d P=0 \quad d Q=\frac{Q}{N}\left(\frac{d f}{f}-\frac{d m}{m}\right)
$$

and (3) becomes

$$
R_{f} \frac{d f}{f}\left(1+\frac{R_{m} / R_{f}}{N}\right)+R_{m} \frac{d m}{m}\left(1-\frac{1}{N}\right)=0
$$


giving, in general, a female bias compared with (5). For the linear tradeoff case $f=1-r$ and $m=r$, and maternal control, (8) solves to give

$$
\begin{aligned}
& r=\frac{N-1}{2 N} \quad(\text { Autosomal control) } \\
& r=\frac{(2 N-1)(N-1)}{N(4 N-1)} \quad(X \text {-control) }
\end{aligned}
$$

obtained by Hamilton (1967 and 1979) and Taylor and Bulmer (1980).

I now mention two or three variations of Hamilton's model to assist in the understanding of local resource competition. First we remove all local mate competition, but retain some inbreeding. Suppose some fixed proportion $q$ of all males are winged and disperse to breed. (Hamilton 1979 offers some evidence for such male dimorphism.) The remaining males each mate with a single female (assuming there are always enough to go round) and the unmated females disperse to mate at random with the winged male population. Then the number of mates per son of a deviant female is

$$
Q^{\prime}=(1-p)+p(f-(1-p) m) / p m=f / m
$$

and is equal to $Q$. So $d Q=0$, there is no local mate competition, and (5) applies. But there is still partial sib-mating, and hence inbreeding.

As another variation suppose the males mate on the patch when small and the mated females continue to grow on the patch until dispersal, so that each patch can only produce a fixed number $M$ of females. Then

$$
Q^{\prime}=\frac{M}{(N-1) m+m^{\prime}} \quad P^{\prime}=\frac{M}{(N-1) f+f^{\prime}}
$$

and (3) becomes

$$
R_{f} \frac{d f}{f}\left(1-\frac{1}{N}\right)+R_{m} \frac{d m}{m}\left(1-\frac{1}{N}\right)=0 .
$$

Cancelling the $1-1 / N$, we get equation (5) again, but we should not say there is no local resource competition, but rather that there is no malefemale asymmetry in this regard. The differentials $d P$ and $d Q$ are both non-zero, but their contributions cancel.

This second variation also provides a model for a finite population (with $M$ breeding sites) and random mating. Of course, eventually, such a population is completely inbred, but the equilibrium sex ratio (in the absence of genetic or production asymmetries) is still $\frac{1}{2}$. Indeed Fisher's classicial sex ratio argument (1930) does not require either an infinite population, or outbreeding, but only random mating.

As a third variation suppose the females are still limited to $M$ per patch, but the males disperse early to breed. So there is no inbreeding and $Q^{\prime}=$ $Q$, but $P^{\prime}$ is still given by (10), and

$$
R_{f} \frac{d f}{f}\left(1-\frac{1}{N}\right)+R_{m} \frac{d m}{m}=0
$$

giving a male bias over (5). Another example of local resource competition in females (in primates) has been described by Clark (1978).

Thus while the magnitude of the local resource competition often depends on the level of inbreeding, the inbreeding should in no way be regarded as causing this component (or any other!) of sex ratio bias.

\section{DISCUSSION}

Nonacs (1985) has reviewed sex allocation phenomenon in ants and has argued that genetic relatedness asymmetries (differences between $R_{f}$ and $R_{m}$ ) appear more significant than local mate competition (differences in $P$ and $Q$ ) in causing sex ratio bias in these populations. A model such as (3) which combines these phenomena into one equation, should make it easier to compare their significance in a quantitative manner. The determination of the amount of inter-colony breeding in these populations is seen to be rather important, as it has an effect on both factors.

The equilibrium equation (3) can be written

$$
R_{f} \frac{d(P f)}{P f}+R_{m} \frac{d(Q m)}{Q m}=0
$$

where $P f$ and $Q m$ are numbers of daughters and sons contributed to the next generation population of mated females. This is an interesting simplification but less useful both practically and conceptually. In practice it is usually more natural to get hold separately of constraints on the production and mating stages of the life cycle. And conceptually, the separation of $P$ and $Q$ from $f$ and $m$ allows us to analyse the important phenomenon of local resource competition.

My assumptions are quite restrictive and it would be nice to generalise the equilibrium equation. Let me mention some possible directions.

(1) More general systems of inbreeding. The terms of (3) all make sense for more general systems than sib-mating, and we can ask if (3) still applies. I would guess yes, but the genetic proof will be more complex. My method of argument 
relies on having "individuals" (they are my mated females) whose offspring do not interact with the offspring of other genetically related individuals during the course of development up to the time of formation of the next generation of individuals. This is more difficult to accomplish with inbreeding that crosses sib boundaries.

(2) Multiple mating. I assumed every mated female had a unique mate, but this assumption can be removed by expanding our notion of type of mutant mated female. For example, if we assume every female has two mates with equal contribution, then a mated female has a ploidy of 6 (or 4 with X-control) and we have many more mutant types. The relatedness of the control to the offspring will now change and must be carefully worked out. Even variable numbers of mates can be handled in this way. So our equation (3) is valid for multiple matings, but care must be taken with $R_{f}$ and $R_{m}$.

(3) Multiple controls. This problem is more difficult. If a large population of control individuals is constructed (e.g., workers) then some assumptions must be made on how different control genotypes will interact. The assumption of "behavioural additivity", that each control individual gets an equal fraction of the reproductive resources and uses its own $r$ on that fraction, leads to our equation, but only really makes sense for the linear tradeoff case $f=1-r, m=r$. In the absence of behavioural additivity things can get complicated. Some recent work is discussed by Bulmer (1983).

(4) Laying workers. I have assumed offspring are made in the standard way: one gamete from each parent. If this assumption is relaxed, equation (3) may fail. The assumption is needed in the argument that identifies the left eigenvector $v$. An example which demonstrates the failure of equation (3) is found in the phenomenon of laying workers in haplodiploidy, under queen control of sex allocation. This has been studied by a number of authors, Trivers and Hare (1976), Charnov (1978) and Taylor (1981), to name but a few. It is equivalent to diploid X-control in which a daughter is permitted to use a portion of the colony's resources to make male offspring of her own. Following Taylor (1981), suppose the daughter gets $\beta$ males for every offspring made by the mated female. With a linear tradeoff, $f=1-r$ and $m=r+\beta$ are the female and male outputs. An examination of the argument for the equation (3) shows that the values of $R_{f}$ and $R_{m}$ used should be those which apply to the extra $d f$ and $d m$ offspring. Thus $R_{f}=R_{m}=$ $1 / 2$. Then assuming outbreeding, equation (3) gives $-1 /(1-r)+1 /(r+\beta)=0$, and $r=(1-\beta) / 2$, whereas the correct answer (Taylor, 1981) is $r=$ $(1 / 2)-(\beta / 4)$.

Finally I emphasise that any solution of (3) must be checked for stability and this is often not easy analytically, and may require reverting to the technicalities of the genetic model and working with the matrix A, something (3) has circumvented. In many applications, a computer check may be reasonable.

Acknowledgements This work was supported by a grant from the Natural Sciences and Engineering Research Council of Canada. I am grateful to the referee for a number of useful comments.

\section{REFERENCES}

BULMER, M. G. 1983. Sex ratio evolution in social hymenoptera under worker control with behavioural dominance. $\boldsymbol{A m}$. Nat., 121, 899-902.

BULMER, M. G. AND TAYLOR, P. D. 1981. Worker-queen conflict and sex ratio theory in social hymenoptera. Heredity, 47, 197-207.

CHARNOV, E. L. 1978. Sex ratio selection in eusocial Hymemoptera. Amer. Natur., 112, 317-326.

CLARK, A. B. 1978. Sex ratio and local resource competition in a prosimian primate. Science, 201, 163-165.

FALCONER, D. S. 1960. Introduction to Quantitative Genetics. The Ronald Press Co., New York.

FISHER, R. A. 1930. The Genetical Theory of Natural Selection. Oxford University Press. Oxford.

GOODMAN, L. A. 1967. On the reconciliation of mathematical theories of population growth. J. Royal Stat. Soc., A/30, $541-553$.

HAMILTON, W. D. 1967. Extraordinary sex ratios. Science, 156, $477-488$.

HAMILTON, W. D. 1979. Wingless and fighting males in fig wasps and other insects. In M. S. Blum and N. A. Blum (eds.) Reproductive Competition and Sexual Selection in Insects. Academic Press, New York.

LI, C. C. 1955. Population Genetics. University of Chicago Press, Chicago, Ill.

SHAW, R. F. AND MOHLER, J. D. 1953. The selective advantage of the sex ratio. Amer. Natur., 87, 337-342.

TAYLOR, P. D. 1981. Sex ratio compensation in ant populations. Evolution, 35, 1250-1251.

TAYLOR, P. D. AND BULMFR, M. G. 1980. Local mate competition and the sex ratio. J. Theor. Biol., 86, 409-419.

TRIVERS, R. L. ANI HARE, H. 1976. Haplodiploidy and the evolution of the social insects. Science, 191, 249-263.

\section{APPENDIX}

\section{Derivation of the equilibrium equation (3)}

We keep track of the frequency of the mutant allele by keeping track of the population of mutant mated females from one generation to the next. Our 
assumptions that the mutation is rare and sibmating is the only source of inbreeding, allow us to assign each mutant mated female to a single mutant mated ancestor one generation back, and this effectively allows us to count mutant genes.

There will be different types of mutant mated females depending on the configuration of mutant alleles. For example, under $\mathrm{X}$-control, there are 5 types which we designate by $(2,1),(2,0),(1,1)$, $(1,0)$ and $(0,1)$, the first and second entries being the number of mutant alleles in mother and father respectively. Denote by $a_{i j}$ the number of mutant mated females of type $i$ which derive from a single type $j$, in one generation, and let $A^{\prime}$ denote the matrix of the $a_{i j}$. The entries of $A^{\prime}$ depend on $r$ and $r^{\prime}$ and the rate of increase of the mutant allele frequency is given by its dominant eigenvalue $\lambda^{\prime}$. If $r^{\prime}=r+d r$, then $A^{\prime}=A+d A$ and $\lambda^{\prime}=\lambda+d \lambda$ and the condition that $r$ be stationary is $d \lambda=0$. This is equivalent to

$$
v d A u=0
$$

where $v$ and $u$ are the left and right dominant eigenvectors of $\boldsymbol{A}$ (Bulmer and Taylor 1981). For example, with $X$-control, $A^{\prime}$ is $5 \times 5$ and, for the case of sib-mating with probability $1 / N$, is given by Taylor and Bulmer (1980).

I now interpret the eigenvectors $v$ and $u$ of $A$. These interpretations are strongly analogous to the interpretations of the right and left eigenvectors of the Leslie matrix in demography as stable age structure and reproductive value vectors (Goodman, 1967). First if $n_{i}$ is the number of mated females of type $i$, then the number after one generation is $\tilde{n}_{i}=\sum a_{i j} n_{j}$. At asymptotic proportions $\tilde{n}_{i}=\lambda n_{i}$ (we assume $r^{\prime}=r$ ), which tells us that $u_{i}$, the frequency of type $i$ mutant mated females is the dominant right eigenvector of $A, A u=\lambda u$.

The key lemma behind the identification of $v$ is that if $r^{\prime}=r$, then at asymptotic proportions every mutant allele in a mated female genotype contributes the same expected number of copies to the next generation. This result, first observed by Hamilton (1967), is verified by direct calculation for each type of control locus. For example, for $\mathrm{X}$-control, an allele on the female side contributes $(f / 2)+(f / m)(m / 2)=f$ copies (every son gets $f / m$ mates) and an allele on the male side contributes $f+0=f$ copies as well. I can now identify $v$. Let $v_{j}$ be the number of mutant alleles in the genotype of a mutant mated female of type $j$. Then the number of mutant alleles contributed to the next generation by a type $j$ mated female is $\sum v_{i} a_{i j}$. If each of her $v_{j}$ alleles make the same number $\lambda$ of copies, then this equals $\lambda v_{j}$, and $v A=\lambda v$. For example, for X-control, with the types listed above, $v=(3,2,2,1,1)$.

These interpretations of the eigenvectors allow me to formulate an important heuristic. Suppose the mutant gene behaves normally $\left(r^{\prime}=r\right)$ and has reached asymptotic proportions $u$. Let it express altered behaviour $\left(r^{\prime} \neq r\right)$ for a single generation. Let $E^{\prime}$ be the expected mutant gene contribution to the next generation of a random mutant mated female. Then

$$
E^{\prime}=v A^{\prime} u
$$

The equilibrium condition (13) can now be written

$$
d E=0 .
$$

This is an important result, both conceptually and computationally. It says that for purposes of writing down a local equilibrium condition, we can regard the mutant allele as if it operates in an altered manner for one generation on!y.

I now derive an alternative expression for $E^{\prime}$. Let $p_{j}$ be the probability that a mutant mated female of type $j$ is deviant, that is, actually expresses the mutation, and makes $\left(f^{\prime \prime}, m^{\prime}\right)$ offspring instead of $(f, m)$. The $p$ vector will depend on the control mechanisms, both locus and genotype, and on dominance assumptions about the control genotype. For example with maternal $\mathrm{X}$-control, we have $p=(1,1,1,1,0)$ for a dominant mutation and $p=(1,1,0,0,0)$ for a recessive mutation. For sister control, $p=\left(1,1,1, \frac{1}{2}, 1\right)$ and $p=$ $\left(1,0, \frac{1}{2}, 0,0\right)$ are the dominant and recessive $p$ vectors respectively. Also let $x_{j}$ and $y_{j}$ be the expected number of mutant alleles in a daughter and son, respectively, of a type $j$ mutant mated female. For example, with X-control, $x(2,1,3,1$, 1) and $y=\left(1,1, \frac{1}{2}, \frac{1}{2}, 0\right)$. Then

$$
\begin{aligned}
E^{\prime}= & \sum_{j}\left(p_{j} P^{\prime} f^{\prime}+\left(1-p_{j}\right) P f\right) x_{j} u_{j} \\
& +\left(p_{j} Q^{\prime} m^{\prime}+\left(1-p_{j}\right) Q m\right) y_{j} u_{j} .
\end{aligned}
$$

Now let $z_{j}$ be the expected number of mutant alleles in a type $j$ control genotype. It is easy to argue that for any haploid control (such as paternal $X$-or $Y$-control), $z_{j}=p_{j}$ and for a diploid control, $z_{j}=p_{j}^{\prime}+p_{j}^{\prime \prime}$ where $p^{\prime}$ and $p^{\prime \prime}$ are the deviant probabilities for a dominant and recessive mutation respectively. Let $E_{d}^{\prime}$ and $E_{r}^{\prime}$ be the dominant and recessive versions of $E^{\prime}$. Note that (for $r^{\prime}=r$ ) $E_{d}=$ $E_{r}=E=P f \bar{x}+Q m \bar{y}$, where $\bar{x}$ and $\bar{y}$ are the average numbers of mutant genes per daughter and son of mutant mated female (the average taken with the type frequencies $u_{i}$ corresponding to the case $r^{\prime}=$ 
$r)$. It follows that

$$
\begin{aligned}
& \left(E_{d}^{\prime}-E_{d}\right)+\left(E_{r}^{\prime}-E_{r}\right) \\
& \quad=\left(P^{\prime} f^{\prime}-P f\right) \sum z_{j} x_{j} u_{j}+\left(Q^{\prime} m^{\prime}-Q m\right) \sum z_{j} y_{j} u_{j} .
\end{aligned}
$$

Now define $\bar{z}=\sum z_{j} u_{j}$ to be the average (with $r^{\prime}=r$ ) number of mutant genes in a control genotype, and notice that

$$
R_{f}=\sum z_{j} x_{j} u_{j} / \bar{z}
$$

and

$$
R_{m}=\sum z_{j} y_{j} u_{j} / \bar{z}
$$

Indeed this follows from the observation that a random mutant allele in a control genotype will be in a type $j$ control with probability $z_{j} u_{j} / \bar{z}$ (assuming, of course, $\hat{r}=r$ ). Then (17) becomes

$$
\begin{aligned}
\left(E_{d}^{\prime}-E_{d}\right)+\left(E_{r}^{\prime}-E_{r}\right)= & \bar{z} R_{f}\left(P^{\prime} f^{\prime}-P f\right) \\
& +\bar{z} R_{m}\left(Q^{\prime} m^{\prime}-Q m\right) \\
= & \bar{z}\left(w^{\prime}-w\right)
\end{aligned}
$$

where $w^{\prime}=R_{f} P^{\prime} f^{\prime}+R_{m} Q^{\prime} m^{\prime}$ is the game-theoretic fitness function introduced earlier. An equilibrium value of $r$ must be stationary for both dominant and recessive mutants, that is, indifferential form ( $\left.r^{\prime}=r+d r\right)$, (15) must hold for both $E_{d}$ and $E_{r}$, and (19) gives $d w=0$ which is equivalent to (3).

\section{The question of dominance}

The key to the simplification of (17) was that for a diploid control, an ESS value of $r$ must be at equilibrium for the introduction of both dominant and recessive mutations. But for any specific assumption about dominance, we can work out the $p_{j}$ and work directly with (17). The question arises of when various dominance assumptions will all give the same equilibrium value of $r$. That is, when is

$$
\frac{\sum p_{j}^{\prime} x_{j} u_{j}}{\sum p_{j}^{\prime} y_{j} u_{j}}=\frac{\sum p_{j}^{\prime \prime} x_{j} u_{j}}{\sum p_{j}^{\prime \prime} y_{j} u_{j}}
$$

where $p^{\prime}$ and $p^{\prime \prime}$ belong to dominant and recessive mutations respectively? If (20) holds then both sides will equal $R_{f} / R_{m}$ and any solution to (3) will give a cancidate for an ESS. If (20) does not hold, the solutions of (3) will not be biologically meaningful and there will not in general (for general $f-m$ tradeoff curves) be an ESS $r$ which is a "pure strategy" for the population. In this case we expect the ESS to be mixed; at equilibrium two or more values of $r$ will be found in the population, and the analysis becomes more difficult.

Note that for the case of autosomal control, $x_{j}=y_{j}$ and (20) holds trivially. For the case of $\mathrm{X}$-control, the $5 \times 5$ matrix $\mathrm{A}$ (Taylor and Bulmer, 1980 ) has right eigenvector

$$
u=\left(2 F^{2}, F(1-F), 2 F(1-F), 2(1-F), 1-F\right)^{T}
$$

and it can be directly verified that (20) holds for both mother and daughter control. Also all types of paternal and fraternal sex linked control are haploid and the problem does not arise.

An example in which (20) fails is obtained with $\mathrm{X}$-control and when the control genotype is a daughter or son each with probability $\frac{1}{2}$. That is, the first offspring is male or female with equal probability, and controls the sex ratio of the brood. For this case the $p$ values are averages of the daughter $p$ values (given above) and the (haploid) son values $p=\left(1,1, \frac{1}{2}, \frac{1}{2}, 0\right)$. Since (20) fails, the equilibrium point given by (3) is meaningless. For such a population there is no single ESS. This example is not of much biological interest because we might expect son-controlled broods to have different sex ratios from daughter-controlled broods. But that is another model. Even if we suppose the control gene cannot tell the sex of its bearer, we still could not have a pure state ESS. 